\title{
EARTH SHADOWS AND THE SEV ANGLE OF' MAP'S LISSAJOUS ORBIT AT L2*
}

\author{
Ariel Edery \\ a.i. solutions, Inc. \\ 10001 Derekwood Lane, Suite 215 \\ Lanham, MD 20706
}

\begin{abstract}
The Microwave Anisotropy Probe (MAP) lannched successfully on June 30, 2001 and is presently in a Lissajous orbit about the Sun-Earth libration point L2. To avoid Earth shadows at L2, the Sun-EarthVehicle (SEV) angle of MAP has to be greater than $0.5 \mathrm{deg}$ for an extended mission of four years. An equation is derived for the SEV angle in terms of the phase angle, frequencies and amplitudes of the Lissajous. The SEV angle is shown to oscillate with a period of 90.4 days within an amplitude envelope of period 13.9 years. A range of phase angles that avoids shadows is identified. MAP's present phase angle is within this range and will avoid shadows for approximately 5.8 years.
\end{abstract}

\section{Introduction}

The Cosmic Microwave Background (CMB) radiation which permeates our universe was originally observed unexpectedly in 1965 by Arno Penzias and Robert Wilson at Bell Laboratories [1]. The CMB radiation has a thermal spectrum with an average temperature of $2.7 \mathrm{Kelvin}$ and is believed to be the leftover radiation from the Big Bang. In 1992, NASA's Cosmic Background Explorer (COBE) [2] satellite provided the first experimental evidence that the microwave glow is not uniformly 2.7 Kelvin, but varies by as much as 100 millionths of a Kelvin above or below the average - evidence of clumps and wrinkles in the very early universe. The follow-up mission to COBE, the Microwave Anisotropy Probe (MAP), will measure the temperature fluctuations of the $\mathrm{CMB}$ radiation with much higher resolution, sensitivity, and accuracy than COBE in the hope of answering unresolved cosmological questions [3].

There exists a point, in the direction opposite the Sun, approximately 1.5 million kilometers from the Earth called the L2 libration point. If a satellite at this point orbits the Sun with the same angular veloc-

\footnotetext{
*work done under NASA contract NASS-01090/Task 126
}

ity as the Earth, its centripetal acceleration is equal to the sum of the Earth and Sun's gravitational acceleration. In other words, the spacecraft remains stationary at $L 2$ in the Sun-Earth rotating frame. At L2, there is no interference from the Earth's atmosphere or radiation belts. Furthermore, one does not have to actively cool the instruments as in the lowEarth COBE mission because the temperature at L2 is already low. With a lunar gravity assist, a satellite can be propelled to $L 2$ at a low Delta-V cost. Once at L2, a Lissajous orbit can be maintained with small stationkeeping maneuvers (approximately $40 \mathrm{~cm} / \mathrm{s}$ of Delta- $\mathrm{V}$ every three months). For all the above stated reasons, a Lissajous orbit about L2 was selected as the mission orbit for MAP.

MAP launched on June 30, 2001 and its trajectory to L2 is shown in Figure 1. It includes three phasing loops around the Earth, a lunar swingby and a Lissajous orbit about L2. There are many possible trajectories to L2, each leading to a different Lissajous. However, the possible choices are greatly reduced by mission requirements. MAP needs to avoid Earth and Lunar shadows as well as maintain communication with NASA's Deep Space Network (DSN). These requirements translate into constraints on the Sun- 
Earth-Vehicle (SEV) angle (the angle $\psi$ shown in Figure 3). For example, Earth shadows constrain the $\mathrm{SEV}$ angle to be greater than $0.5 \mathrm{deg}$. It is therefore imperative to predict how the SEV angle varies with time, which is the main goal of this paper.

We begin by solving for quantities that enter into the calculation of the SEV angle. From the periodic solutions of the linearized equations of motion we determine that the $x$ and $y$ components oscillate with the same frequency $\omega_{x y}$ (period of 0.4861 years) and the $z$ component oscillates with $\omega_{z}$ (period of 0.5023 years). The two periods are slightly different, leading to a Lissajous orbit about L2. We then derive an equation for the SEV angle as a function of time. The equation reveals that the SEV angle exhibits a beat pattern: the amplitude of oscillations does not remain constant and oscillates with its own frequency - called the beat frequency. It is shown that the two frequencies that govern the $S E V$ angle are not $\omega_{x y}$ and $\omega_{z}$ but their sum $\omega^{+}=\omega_{x y}+\omega_{z}$ and difference $\omega^{-}=\omega_{x y}-\omega_{z}$. The SEV angle oscillates with $\omega^{+}$ equivalent to a period of 90.3 days and the amplitude oscillates with $\omega^{-}$equivalent to a much longer period of 13.9 years. Simply put, the SEV angle oscillates within an envelope of period 13.9 years.

A Lissajous is said to be opening when the SEV envelope minimum is increasing. Otherwise it is said to be closing. The amplitude oscillations cause the Lissajous to alternate between opening and closing. We show that MAP must enter the Lissajous while it is opening if it is to avoid Earth shadows for an extended mission of four years. One of the parameters that describes a Lissajous is the phase angle $\phi$ which is related to the initial conditions of the $z$ component of the Lissajous (see equation (24)). We show that the opening or closing of a Lissajous is dependent on its phase angle: when the phase angle is between $\pi$ and $\pi / 2$, the Lissajous is opening. MAP's phase angle is presently in this range and we show that it will avoid Earth shadows for approximately 5.8 years starting from the first $\mathrm{xz}$ plane crossing.

\section{The Sun-Earth Libration Point L2}

In this section we outline briefly how to determine numerically and analytically the location of the libra-
Figure 1: Trajectory to L2

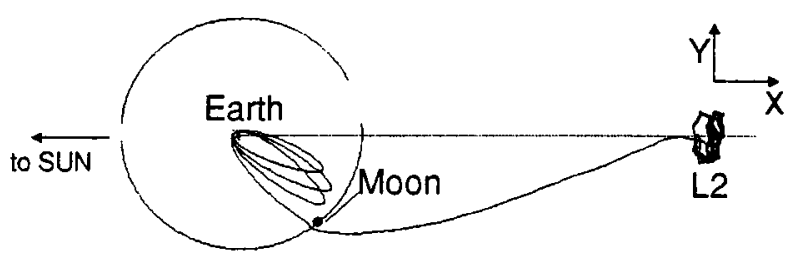

tion point L2 for the Sun-Earth/Moon system (for more details on the L2 libration point we refer the reader to $[4,5,6])$. We work in dimensionless units where the Sun-Earth distance is 1, the mass of the Sun is $M_{S}=1-\mu$ and the mass of the Earth + Moon is $M_{E}=\mu$ so that $M_{S}+M_{E}=1$. The latest value of $M_{S} / M_{E}$ is 328900.56 with an uncertainty of \pm 0.02 [7]. The value of $\mu=1 /\left(1+M_{S} / M_{E}\right)$ for the SunEarth/Moon system is therefore $3.0404234 \times 10^{-6}$. The most convenient frame in which to study motion about L2 is the Sun-Earth rotating frame where the $x$ axis points along the Sun-to-Earth line (see Figure 1) and the $y$ axis is in the ecliptic plane perpendicular to $x$ (pointing in the direction of the Earth's motion about the Sun). The $z$ axis completes the right-handed coordinate system. The distance $r$ from Earth to L2 is governed by the quintic equation [5]:

$$
r^{5}+(3-\mu) r^{4}+(3-2 \mu) r^{3}-\mu r^{2}-2 \mu r-\mu=0
$$

Using the MATLAB root finder with $\mu=3.0404234 \times$ $10^{-6}$, the real solution to (1) is $r=0.010078240$ (this value is in agreement with the value quoted in [8] which contains a comprehensive set of libration-point parameters). In 1826, Abel proved that the solution to the general quintic cannot be written as a finite formula involving radicals. However, for $\mu \ll 1$ an analytical approximation to (1) can readily be obtained and yields results close to the numerical one (the case $\mu \ll 1$ applies to all Sun-Planet libration points). To find an analytical solution it is convenient to rewrite the quintic equation (1) in the following form

$$
f(r)=\frac{r^{3}\left(3-2 \mu+(3-\mu) r+r^{2}\right)}{(1+r)^{2}}=\mu .
$$


The function $f(r)$ is a monotonic increasing function, has no upper bound and has a minimum value of zero (which occurs at $r=0$ ). Therefore, the equation $f(r)=\mu$ must have one and only one real solution. Furthermore, if $\mu \ll 1$ then $r$ lies in the range $0<r \ll 1$. Expanding $f(r)$ yields $3 r^{3}+\mathcal{O}\left(r^{3}\right)$. Keeping only the first term in this expansion yields the following analytical approximation to (2)

$$
r \approx(\mu / 3)^{1 / 3} .
$$

The above analytical approximation reveals that the L2 distance grows as the cube root of $\mu$ for $\mu \ll 1$. Let us now compare the analytical with the numerical solution for the Sun-Earth/Moon system. For $\mu=3.0404234 \times 10^{-6}$, the analytical approximation (3) yields $r=0.010044715$ which differs from the numerical solution of $r=0.010078240$ by $0.33 \%$. The analytical approximation (3) is therefore very accurate for small $\mu$. Note that we do not quote $r$ as 0.010 but keep eight significant figures. We will show later that the difference between 0.010 and 0.010078240 is significant for the calculation of the frequencies governing the Lissajous and the SEV angle. However, most other calculations in this paper are not as sensitive and using $r=0.010$ works well.

\section{Earth Shadows and the SEV angle}

During MAP's orbit about L2, one of the requirements of the mission was for the satellite to avoid umbral and penumbral shadows. The umbral and penumbral shadows are depicted in Figure 2. No direct sunlight reaches the umbral region and therefore the MAP satellite must certainly avoid this region. It turns out that the umbral shadow falls just short of reaching $\mathrm{L} 2$ and hence causes no problems for the MAP satellite. Let $x$ be the distance from the center of the Earth to where the umbral shadow terminates. Let $R_{E}$ be the radius of the Earth, $R_{S}$ be the radius of the Sun and the Sun-Earth distance be equal to 1 . The geometry leads to the following equality

$$
\frac{x}{1+x}=\frac{R_{E}}{R_{S}}=\frac{6378}{695990}=9.16 \times 10^{-3}
$$

whose solution is $x=0.00924$. The length $x$ of the umbral shadow is less than the Earth-L2 distance of
Figure 2: Umbral and Penumbral Shadows

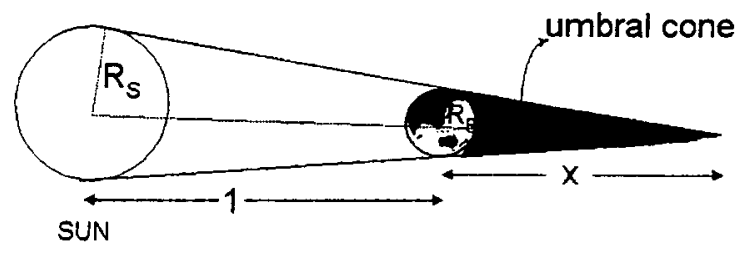

(not to scale)

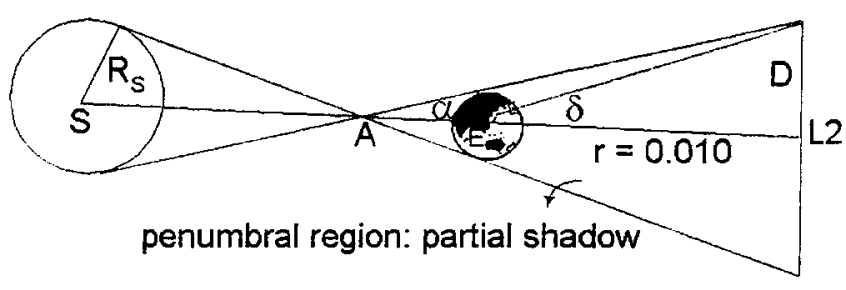

$r=0.010$ and therefore MAP does not encounter umbral shadows. Note that this result is valid for any Sun-Earth distance on the Earth's elliptical journey around the Sun. This is true because the values of $x$ and $r$ are in dimensionless units and are independent of the Sun-Earth distance. It is clear from Figure 2 that the SEV angle $\delta$ must be above a certain value to avoid the penumbral shadow. The penumbral shadow is a partial shadow and therefore less severe than the umbral shadow. Nonetheless, it was considered desirable to avoid this region altogether. We now determine how it constrains the SEV angle of MAP. Let SA denote the distance from the Sun to point $A$ and let $A E$ denote the distance from $A$ to the Earth. Again, the geometry leads to the following equality

$$
\frac{A E}{S A}=\frac{R_{E}}{R_{S}}=9.16 \times 10^{-3}
$$

whose solution is $\mathrm{AE}=9.08 \times 10^{-3}$ (where $A E+S A=$ 1). The mean Sun-Earth distance is $1.495 \times 10^{8} \mathrm{~km}$ so that the Earth radius $R_{E}$ in dimensionless units is $6378 / 1.495 \times 10^{8}=4.26 \times 10^{-5}$. The angles $\delta$ and $\alpha$ are small so that $\delta \approx D / r$ and $\alpha \approx D /(r+A E)$. The angle $\alpha$ is equal to $\sin ^{-1}\left(R_{E} / A E\right)=4.69 \times 10^{-3} \mathrm{rad}$. 
The angle $\delta$ is therefore

$$
\delta \approx \frac{\alpha(r+A E)}{r}=8.94 \times 10^{-3} \mathrm{rad}=0.51 \mathrm{deg} .
$$

where $r=0.010$ was used. As long as MAP maintains an SEV angle above 0.5 deg during its oscillations about L2 it avoids Earth shadows: both umbral and penumbral.

\section{Frequencies of Lissajous Orbit}

In this section we solve the linear differential equations governing motion at L2. In particular, we obtain the frequencies and the relationship between the $x$ and $y$ amplitudes that govern the Lissajous orbit and enter into the calculation of the SEV angle. Consider the point L2 in Figure 1 to be at $(0,0,0)$. Let $r_{1}$ be the Sun-L2 distance and $r$ be the Planet-L2 distance. In dimensionless units $r_{1}-r=1$. The set of linearized differential equations governing the $x, y$ and $z$ motion about L2 are [5]:

$$
\begin{aligned}
\ddot{x}-2 \dot{y} & =x(1+2 D) \\
\ddot{y}+2 \dot{x} & =y(1-D) \\
\ddot{z} & =-D z
\end{aligned}
$$

where

$$
D=\frac{1-\mu}{r_{1}^{3}}+\frac{\mu}{r^{3}} .
$$

For the Sun-Earth/Moon system, $\mu=3.0404234 \times$ $10^{-6}, r=0.010078240, r_{1}=1.010078240$ and (10) yields $D=3.9405$. Had we used $r=0.010$ and $r_{1}=$ 1.010 the value of $D$ would have been 4.011, which is a significant change from 3.9405. For the rest of the calculations in this paper we do not need to keep results to this accuracy, which is why $D$ is quoted to only five instead of eight significant digits. The equation governing the $z$ motion is easily solved and yields $z=A_{z} \sin \left(\omega_{z} t+\phi\right)$ where $A_{z}$ is the amplitude, $\phi$ is the phase angle and

$$
\omega_{z}=\sqrt{D}=\sqrt{3.9405}=1.9851
$$

is the frequency. The period of the $z$ motion is therefore $1 / 1.9851=0.50375$ years $(\approx 6$ months $)$. We now seek periodic solutions to the coupled $x$ and $y$ equations (7) and (8). Let $y=A_{y} \sin \left(\omega_{x y} t+\phi_{x y}\right)$. Let the xz plane crossing $(y=0)$ occur at $t=0$ so that $\phi_{x y}=0$ or $\pi$ (depending on whether $\dot{y}$ is positive or negative at $y=0$ ). For the MAP mission, the lunar flyby occurs in the negative $y$ region and the satellite is then propelled towards $y=0$ so that $\dot{y}>0$ at $y=0$. Hence, $\phi_{x y}=0$. Therefore, $y=A_{y} \sin \left(\omega_{x y} t\right)$, $\dot{y}=\omega_{x y} A_{y} \cos \left(\omega_{x y} t\right)$ and $\ddot{y}=-\omega_{x y}^{2} A_{y} \sin \left(\omega_{x y} t\right)$. We can readily solve the $x$ and $y$ coupled equations if we let $x=A_{x} \cos \left(\omega_{x y} t\right)$. Equation (7) yields

$$
\frac{A_{x}}{A_{y}}=\frac{2 \omega_{x y}}{-\omega_{x y}^{2}-1-2 D}
$$

and the (8) yields

$$
\frac{A_{x}}{A_{y}}=\frac{-\omega_{x y}^{2}-1+D}{2 \omega_{x y}} .
$$

Equating (12) with (13) one obtains the quartic equation

$$
\omega_{x y}^{4}+(D-2) \omega_{x y}^{2}+\left(1+D-2 D^{2}\right)=0 .
$$

Using the MATLAB root finder, the positive real solution to (14) with $D=3.9405$ is

$$
\omega_{x y}=2.0570 \text {. }
$$

The period of the $x$ and $y$ motion is therefore $1 / 2.0570=0.4861$ years, i.e. approximately 5.83 months. Substituting $\omega_{x y}=2.0570$ into either (12) or (13) yields $A_{x} / A_{y}=-0.3138$. Therefore the periodic solutions to $(7),(8)$ and (9) are:

$$
\begin{aligned}
& x=-0.3138 A_{y} \cos (2.0570 t) \\
& y=A_{y} \sin (2.0570 t) \\
& z=A_{z} \sin (1.9851 t+\phi) .
\end{aligned}
$$

Note that when $x$ and $y$ complete one cycle, $z$ has not quite completed its own cycle because the frequency in the $x y$ and $z$ directions differ slightly (2.0570 versus 1.9851). Therefore, the motion at L2 is only quasiperiodic even though the components $x, y$ and $z$ are each periodic functions. Such a trajectory governed by two different frequencies is called a Lissajous orbit in contrast to a halo orbit where the two frequencies are equal. Later we will see that the slight difference 
in the $x y$ and $z$ frequencies is actually the reason why the SEV angle exhibits a beat pattern.

The amplitudes $A_{y}$ and $A_{z}$ and the phase angle $\phi$ are determined by the initial conditions: $\dot{y}, \dot{z}, x$ and $z$ at $t=0$. Note that the $x$ and $y$ equations (16) and (17) are coupled so that when $y=0$, the $x$-velocity $\dot{x}=0$. In other words, to have quasi-periodic motion at L2, the $x$-velocity of the satellite (i.e. the velocity along the Sun-Earth line) must be close to zero when it crosses the $\mathrm{xz}$ plane at $y=0$. If this condition is not met, the satellite may not enter a quasi-periodic orbit and can easily escape from L2. In fact, the libration point L2 is not a point of stable equilibrium: besides the periodic solutions there exist exponential solutions for the $x$ and $y$ motion. If one substitutes $x=C_{1} e^{k t}$ and $y=C_{2} e^{k t}$ into the $x$ and $y$ equations (7) and (8), one obtains the same quartic as in (14) except that one chooses the imaginary instead of the real solutions. This yields $k= \pm 2.484$ and $C_{1}=$ $\mp 1.834 C_{2}$. The most general solution to the linear differential equations (7), (8) and (9) is:

$$
\begin{aligned}
x= & -0.3138 A_{y} \cos \left(2.0570 t+\phi_{x y}\right) \\
& -1.834 C_{1} e^{2.484 t}+1.834 C_{2} e^{-2.484 t}(19) \\
y= & A_{y} \sin \left(2.0570 t+\phi_{x y}\right)+C_{1} e^{2.484 t} \\
& +C_{2} e^{-2.484 t} \\
z= & A_{z} \sin (1.9851 t+\phi) .
\end{aligned}
$$

The six constants in (19), (20) and (21) can be expressed in terms of the initial conditions at the first $\mathrm{xz}$ plane crossing $(\mathrm{y}=0): x=x_{0}, v_{x}=v_{x_{0}}, v_{y}=v_{y_{0}}$, $z=z_{0}$ and $v_{z}=v_{z_{0}}$. The constants are:

$$
\begin{aligned}
\tan \phi_{x y} & =\frac{v_{x_{0}}}{4.316 x_{0}+3.187 v_{y_{0}}} \\
A_{y} & = \\
& 0.192 \sqrt{v_{x_{0}}^{2}+\left(4.316 x_{0}+3.187 v_{y_{0}}\right)^{2}} \\
\tan \phi= & \frac{1.9851 z_{0}}{v_{z_{0}}} \\
A_{z}= & \sqrt{0.253 v_{z 0}^{2}+z_{0}^{2}} \\
C_{1}= & -0.0961 v_{x 0}-0.3436 x_{0} \\
& -0.0524 v_{y 0}
\end{aligned}
$$

Figure 3: SEV Angle

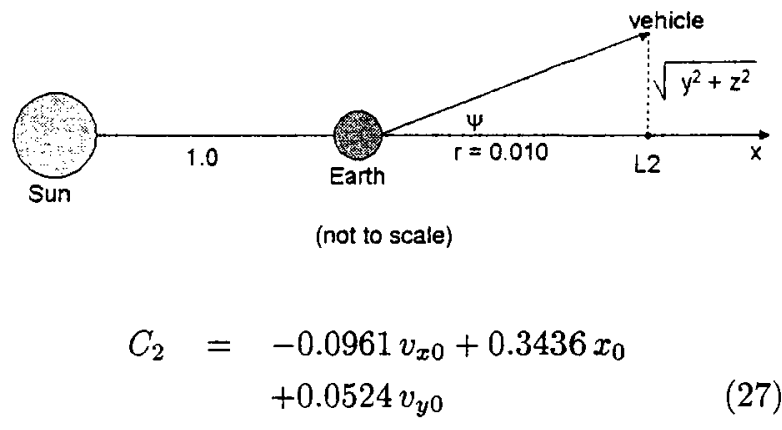

The periodic solutions (16), (17) and (18) are recovered when the exponential solutions are suppressed i.e. $C_{1}=C_{2}=0$ in (19) and (20). If $C_{1}=C_{2}=0$ then it follows from (26) and (27) that $v_{x_{0}}=0$. This implies that $\phi_{x y}$ given by (22) is zero. So as previously mentioned, $v_{x}$ must be close to zero at the $\mathrm{xz}$-plane crossing to obtain a Lissajous orbit at L2.

To summarize, L2 is an unstable point that can support a quasi-periodic orbit. The $x, y$ and $z$ equations of motion have both exponential and periodic solutions. The exponential solutions are largely suppressed if the $x$-velocity at the $\mathrm{xz}$ plane crossing is zero. The frequency of the periodic oscillations in the $x$ and $y$ direction is $\omega_{x y}=2.0570$ and in the $z$ direction is $\omega_{z}=1.9851$ (equivalent to a period of approximately 6 months in both cases). The two frequencies are slightly different and the resultant motion is a Lissajous orbit.

\section{Beat Pattern of Sun-Earth-Vehicle (SEV) Angle}

In this section we derive an expression for the SunEarth-Vehicle (SEV) angle as a function of time for oscillatory motion at L2. The expression is written in terms of two frequencies, $\omega^{+}$and $\omega^{-}$, which are the sum and difference of $\omega_{x y}$ and $\omega_{z}$ respectively. The frequency $\omega^{+}$describes the oscillations of the SEV angle while $\omega^{-}$, called the beat frequency, corresponds to the oscillations of the amplitude envelope. The SEV angle $\psi$ is given by

$$
\tan \psi=\frac{\sqrt{y^{2}+z^{2}}}{r}
$$


where $r=0.010$ is the Earth-L2 distance (see Figure 3 ). To determine the SEV angle $\psi$, we need to calculate $y^{2}+z^{2}$. For periodic motion about L2, $y$ and $z$ are given by (17) and (18) i.e.

$$
y=A_{y} \sin \left(\omega_{x y} t\right) \quad ; \quad z=A_{z} \sin \left(\omega_{z} t+\phi\right)
$$

and the sum of their squares yields

$$
y^{2}+z^{2}=A_{y}^{2} \sin ^{2}\left(\omega_{x y} t\right)+A_{z}^{2} \sin ^{2}\left(\omega_{z} t+\phi\right) .
$$

It is clear from (30) that $y^{2}+z^{2}$, and hence the SEV angle, has oscillations. However, it is not immediately apparent from (30) what the frequency is of those oscillations. It is not either $\omega_{x y}$ or $\omega_{z}$ but some combination of the two. It is therefore worthwhile to rewrite equation (30) in a different form where the frequencies governing the SEV angle are apparent. The first step is to rewrite (30) as:

$$
\begin{aligned}
y^{2}+z^{2} & =\frac{A_{y}^{2}+A_{z}^{2}}{2}\left(\sin ^{2}\left(\omega_{x y} t\right)+\sin ^{2}\left(\omega_{z} t+\phi\right)\right) \\
+ & \frac{A_{y}^{2}-A_{z}^{2}}{2}\left(\sin ^{2}\left(\omega_{x y} t\right)-\sin ^{2}\left(\omega_{z} t+\phi\right)\right) . \quad(31)
\end{aligned}
$$

Using the trig identities

$$
\sin ^{2} \theta_{1}-\sin ^{2} \theta_{2}=\sin \left(\theta_{1}+\theta_{2}\right) \sin \left(\theta_{1}-\theta_{2}\right)
$$

and

$$
\cos ^{2} \theta_{1}-\sin ^{2} \theta_{2}=\cos \left(\theta_{1}+\theta_{2}\right) \cos \left(\theta_{1}-\theta_{2}\right)
$$

one obtains

$$
\begin{aligned}
& \sin ^{2}\left(\omega_{x y} t\right)+\sin ^{2}\left(\omega_{z} t+\phi\right)=1- \\
& \quad \cos \left(\left(\omega_{x y}+\omega_{z}\right) t+\phi\right) \cos \left(\left(\omega_{x y}-\omega_{z}\right) t-\phi\right)(34)
\end{aligned}
$$

and

$$
\begin{aligned}
& \sin ^{2}\left(\omega_{x y} t\right)-\sin ^{2}\left(\omega_{z} t+\phi\right)= \\
& \quad \sin \left(\left(\omega_{x y}+\omega_{z}\right) t+\phi\right) \sin \left(\left(\omega_{x y}-\omega_{z}\right) t-\phi\right.
\end{aligned}
$$

Define the following quantities:

$$
\omega^{+} \equiv \omega_{x y}+\omega_{z} \quad ; \quad \omega^{-} \equiv \omega_{x y}-\omega_{z}
$$

Substituting (34) and (35) into (31) yields

$$
\begin{aligned}
y^{2}+ & z^{2}= \\
& \frac{\left(A_{y}^{2}+A_{z}^{2}\right)}{2}\left(1-\cos \left(\omega^{+} t+\phi\right) \cos \left(\omega^{-} t-\phi\right)\right) \\
+ & \frac{\left(A_{y}^{2}-A_{z}^{2}\right)}{2} \sin \left(\omega^{+} t+\phi\right) \sin \left(\omega^{-} t-\phi\right) .
\end{aligned}
$$

Equation (37) is our final expression for $y^{2}+z^{2}$. It is now apparent that the two frequencies that govern the oscillations of $y^{2}+z^{2}$ are $\omega^{+}$and $\omega^{-}$which are the sum and difference of $\omega_{x y}$ and $\omega_{z}$ respectively. The SEV angle $\psi$ given by (28) is proportional to the square root of $y^{2}+z^{2}$ and therefore oscillates with the same frequencies as $y^{2}+z^{2}$. For the Sun-Earth L2 libration point, $\omega_{x y}=2.0570$ and $\omega_{z}=1.9851$. Substituting these values into (36) yields

$$
\omega^{+}=4.0421 ; \quad \omega^{-}=0.0719
$$

corresponding to periods of

$$
T^{+}=\frac{1}{4.0421} \text { years }=90.36 \text { days }
$$

and

$$
T^{-}=\frac{1}{0.0719} \text { years }=13.9 \text { years } .
$$

A plot of the SEV angle versus time calculated using (37) and (28) is shown in Figure 4. The way to read this figure is as follows: time increases to the right with the location of $t=0$ situated at the appropriate phase angle on the $x$-axis. For example, if a Lissajous has a phase angle of $\pi / 4$, the SEV angle begins at that location and then moves to the right. Note that the amplitude envelope of the SEV angle does not remain constant but varies periodically. A plot like Figure 4 with an oscillating amplitude envelope is said to exhibit a beat pattern and the frequency of the oscillations is called the beat frequency. The regular oscillations correspond roughly to $\omega^{+}$and the time between adjacent peaks is $\approx 90.36$ days. The envelope oscillations correspond roughly to $\omega^{-}$(the beat frequency) and are represented by the dashed line. These oscillations have a period of $\approx 13.9$ years. In Figure 4, peak to peak on the dashed curve represents one half of the period i.e. 6.95 years. This is 
Figure 4: Closing and Opening Lissajous

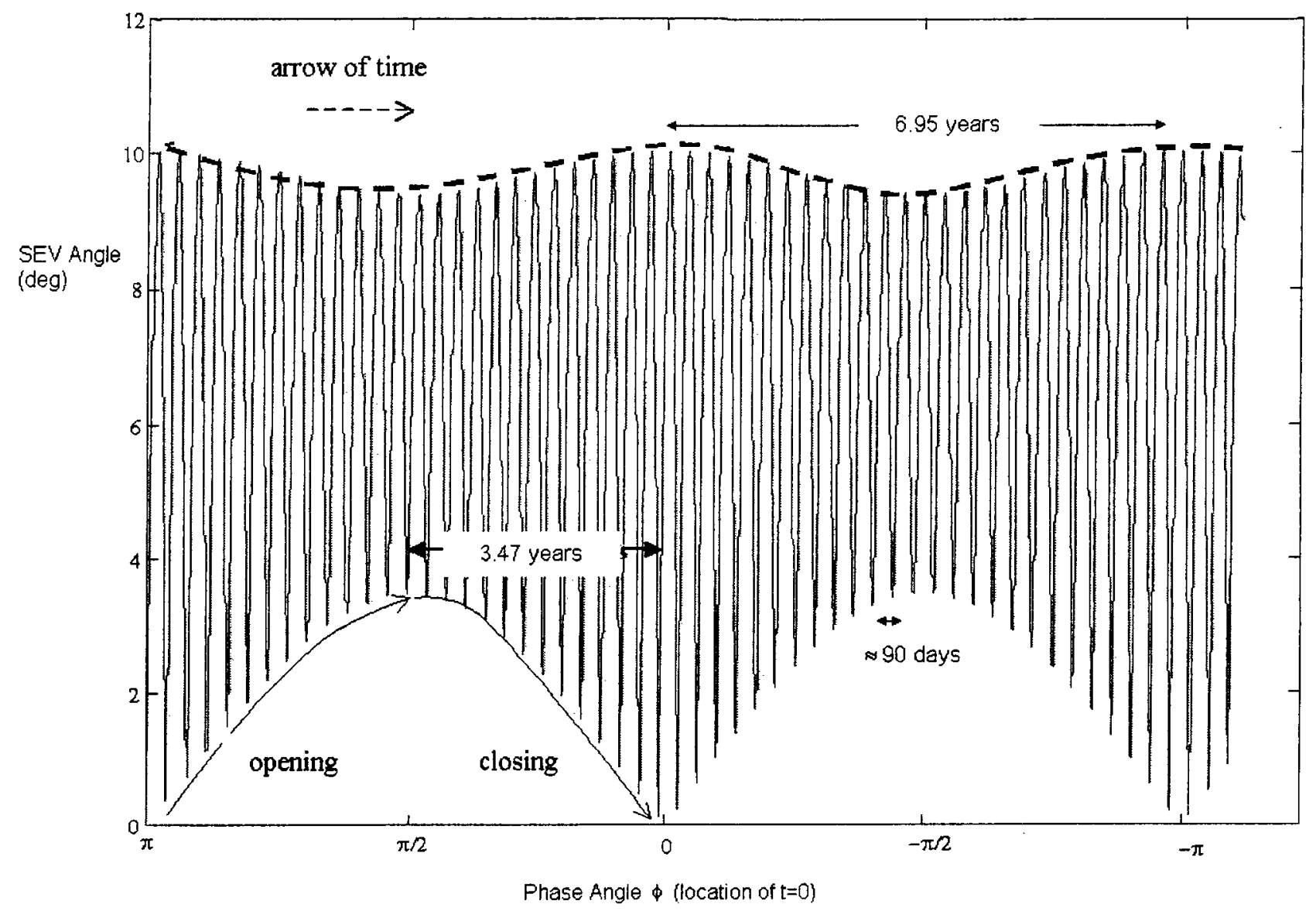


because the SEV angle is always a positive quantity and repeats itself every half cycle. The sine/cosine functions undergo a full cycle every 13.9 years and are positive for half a cycle and negative for half a cycle. The Lissajous is in a different orientation in each of the two half cycles but the value of the SEV angle, being always positive, does not distinguish between these two half cycles.

The phase angle $\phi$ of the Lissajous determines whether it is opening or closing at $t=0$. An important result stemming from equation (37) is that the Lissajous is opening when the phase angle is between $\pi$ and $\pi / 2$ and closing between $\pi / 2$ and 0 (see Figure 4). It takes the Lissajous a maximum of $(13.9 / 4)=3.47$ years to close. In other words, when the lissajous is closing, the SEV angle reaches a value of zero in less than 3.47 years (see Figure 4 ). The extended mission of MAP is to last four years. This is greater than the maximum closing time of 3.47 years. We have previously seen that the SEV angle should be greater than 0.5 degrees to avoid penumbral shadows. Therefore, if the MAP satellite enters the Lissajous while it is closing, it will reach a zero SEV angle before the four year mission is complete and hence encounter penumbral shadows. In other words, if MAP enters a closing Lissajous it is sure to cross the 0.5 degree line before four years are completed. It is therefore crucial that the MAP satellite enter an opening Lissajous. This implies that the phase angle of MAP must be between $\pi$ and $\pi / 2$.

We briefly digress here to discuss some of the symmetry properties of the SEV angle. The equation for $y^{2}+z^{2}$ given by either (30) or (37) is invariant under two sets of transformations:

$$
\begin{array}{rl}
t \rightarrow-t & t \rightarrow-t \\
\phi \rightarrow-\phi & \phi \rightarrow \pi-\phi
\end{array}
$$

Therefore, if we propagate backwards with a new phase angle given by either of the two transformations in (41), the SEV angle (28) does not change. For example, the SEV angle for a Lissajous with phase angle equal to $\pi / 4$ when propagated forwards is indistinguishable from one that has a phase angle of $-\pi / 4$ or $3 \pi / 4$ propagated backwards. There are phase angles however, that play a dual role where propagating forwards or backwards yields the same result. These phase angles are integer multiples of $\pi / 2$ : they do not change under either of the two phase angle transformations (41) (e.g. $\pi / 2$ under $\phi \rightarrow \pi-\phi$ yields $\pi / 2$ and $\pi$ under $\phi \rightarrow-\phi$ yields $-\pi$ which is the same angle). For these, the SEV angle is symmetric under time-reversal. Note that the plot in Figure 4 is mirror-symmetric at phase angles that are integer multiples of $\pi / 2$. If one starts at those phase angles it does not matter whether you move to the right (propagate forwards) or move to the left (propagate backwards).

\section{MAP's Lissajous}

MAP first crossed the $\mathrm{xz}$ plane at L2 on January 2, 2002 at 8:50 UTC time. We consider this to be the start of the Lissajous orbit, i.e. we set $t=0$ when $y=0$. There are altogether five parameters that enter (37) for $y^{2}+z^{2}$ (from which the SEV angle is calculated). The five parameters are the two frequencies $\omega^{+}$and $\omega^{-}$, the two amplitudes $A_{y}$ and $A_{z}$ and the phase angle $\phi$. The value of the frequencies have previously been obtained and are $\omega^{+}=4.0421$ and $\omega^{-}=0.0719$. These frequencies are determined only by the SunEarth/Moon geometry and masses and are therefore independent of the particular Lissajous at L2. The other three parameters are obtained by fitting the ephemeris file of the actual MAP trajectory to the $x, y$ and $z$ equations of motion (16),(17) and (18). Our least-squares fit to the actual MAP ephemeris data yield the following results: $A_{y}=5.88 \times 10^{-4}$, $A_{z}=1.58 \times 10^{-3}$ and $\phi=2.88 \mathrm{rad}$. Note that $\phi=2.88 \mathrm{rad}$ is between $\pi$ and $\pi / 2 \mathrm{rad}$, and this implies that the MAP Lissajous should be opening at $t=0$. The values of $A_{y}, A_{z}$ and $\phi$ were then independently verified with equations (23), (24) and (25). In other words, we extracted the values of $x_{0}, z_{0}, v_{x_{0}}$, $v_{y_{0}}$ and $v_{z_{0}}$ from the ephemeris data of the MAP Lissajous when $y$ was zero. We substituted these values into equations (23),(24) and (25) and calculated $A_{y}=5.83 \times 10^{-4}, A_{z}=1.59 \times 10^{-3}$ and $\phi=2.91$ rad. The two methods therefore agree to within $1 \%$.

The values of $\omega^{+}, \omega^{-}, A_{y}, A_{z}$ and $\phi$ were then substituted in (37) and the SEV angle was calculated via equation (28). A plot of the predicted SEV angle of 
Figure 5: Predicted SEV of MAP

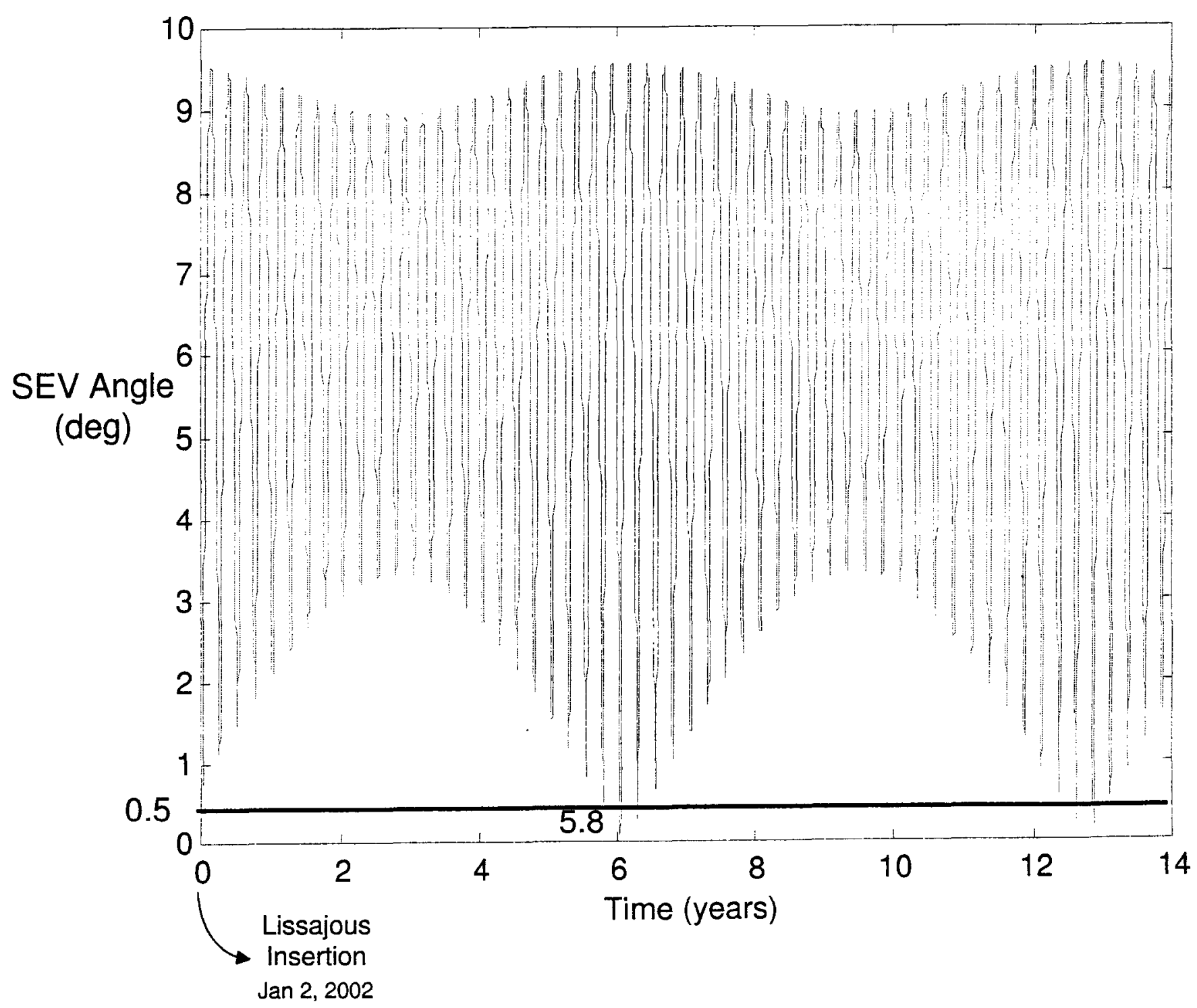


MAP is shown in Figure 5. As predicted, the Lissajous is opening at $t=0$ (corresponding to January 2, 2002 8:50 UTC). Note that the SEV angle starts above the $0.5 \mathrm{deg}$ line and crosses it 5.8 years later. This implies that the MAP satellite will avoid Earth shadows for the extended mission of four years.

A plot of the observed/true SEV angle of MAP starting on January 2, 2002 8:50 UTC and extending to the fourth $\mathrm{xz}$ plane crossing predicted to occur on September 26, 2002 20:16 UTC is shown in Figure 6. This is a time span of 0.733 years. The SEV angle in this case is taken directly from the MAP trajectory, which includes the latest orbit determination solution and the updated coefficient of reflection for the modeling of the solar radiation pressure (important when extending the trajectory to September 26, 2002). Compare the "observed" plot, Figure 6, to the "predicted" plot, Figure 7, over the same time period of 0.733 years. The plots are almost identical in shape and in value. The observed plot has an initial SEV angle of $2.47^{\circ}$, an SEV angle of $9.56^{\circ}$ at the first peak and a peak to peak time of 91.3 days. The predicted plot has an initial SEV angle of $2.35^{\circ}$, an SEV angle of $9.52^{\circ}$ at the first peak and a peak to peak time of 91.6 days. Both peak to peak times are very close to the value of $T^{+}=90.4$ days. The two plots are almost identical even though the predicted plot does not include the effects of solar radiation pressure.

The small difference between the numerical value of 91.6 days obtained by plotting (37) and $T^{+}=90.4$ days is due to the fact that the $\omega^{+}$oscillations are slightly perturbed by the $\omega^{-}$oscillations in (37). The effect is not very pronounced because the $\omega^{+}$frequency is 56 times larger than the $\omega^{-}$frequency. One can easily compute numerically where the crests or troughs of the SEV angle occur by setting the derivative with respect to time of equation (30) to zero, yielding

$$
\omega_{x y} A_{y}^{2} \sin \left(2 \omega_{x y} t\right)+\omega_{z} A_{z}^{2} \sin \left(2\left(\omega_{z} t+\phi\right)\right)=0 .
$$

Substituting $\omega_{x y}=2.057, \omega_{z}=1.9851, A_{y}=$ $5.88 \times 10^{-4}, A_{z}=1.58 \times 10^{-3}$ and $\phi=2.88 \mathrm{rad}$ into (42), the first four positive numerical solutions are $t=0.0184,0.1438,0.2693$ and 0.3948 years. The first and third solutions correspond to the first two troughs (minima) in Figure 7 and the second and fourth solutions correspond to the first two crests (maxima) in Figure 7. Therefore, the peak to peak time is $0.3948-0.1438=0.251$ years (91.6 days). This is only a $1.3 \%$ difference with $T^{+}=90.4$ days so that for all intents and purposes $\omega^{+}$can be viewed as the frequency of the regular SEV oscillations.

\section{Conclusion}

We conclude by summarizing the main results of this paper. We derived equation (37) for $y^{2}+z^{2}$ from which the SEV angle was calculated via equation (28). Equation (37) was cast in a form that makes apparent the frequencies governing the SEV oscillations. We predicted that the regular SEV oscillations have a period of $T^{+}=90.4$ days and that the amplitude envelope has a period of $T^{-}=13.9$ years. The period of the SEV oscillations observed on MAP is 91.3 days. The predicted value of 90.4 days differs from this observed value by $1 \%$. It is worth noting that the periods $T^{+}=90.4$ days and $T^{-}=13.9$ years are results which apply to all Lissajous orbits about $L 2$. In contrast, the amplitudes and phase angle depend on the particular Lissajous in question.

We showed that a Lissajous closes in 3.47 years so that MAP must enter an opening Lissajous if it is to avoid Earth shadows for an extended mission of four years. Through equation (37) we determined that a Lissajous is opening when the phase angle $\phi$ is between $\pi$ and $\pi / 2$ and closing when the phase angle is between $\pi / 2$ and 0 . The phase angle of MAP was found to be $2.88 \mathrm{rad}$. Therefore MAP entered an opening Lissajous.

The phase angle $\phi$ and the amplitudes $A_{y}$ and $A_{z}$ of the MAP Lissajous were obtained by fitting the ephemeris data of the MAP trajectory to the $x, y$ and $z$ equations of motion $(16),(17)$ and (18). These three Lissajous parameters were substituted into equation (37) for $y^{2}+z^{2}$ and the SEV angle was calculated via equation (28). A plot of the SEV angle versus time was made. In the last section we showed that this "predicted" SEV plot matched well with the "observed" SEV plot of MAP. This shows that the derived equation (37) for $y^{2}+z^{2}$ is a reliable equation for making predictions on the SEV angle. 
In our shadow analysis, we showed that a spacecraft orbiting at L2 enters Earth shadows if it reaches an SEV angle below 0.5 deg. By extending the "predicted" plot of MAP's SEV angle, we found that it dips below 0.5 deg after 5.8 years(i.e. 5.8 years after the spacecraft first crosses the $\mathrm{xz}$ plane). Therefore, MAP should avoid Earth shadows for approximately 5.8 years. This is well within the requirement that MAP should avoid Earth shadows for its extended mission of four years. It is worth noting that the small stationkeeping maneuvers $(\approx 40 \mathrm{~cm} / \mathrm{s})$ that are performed approximately every three months simply maintain the Lissajous in its current orbit and hardly change its parameters. Therefore, the stationkeeping maneuvers do not perturb in any significant way the 5.8 year prediction.

Figure 6: Observed/True SEV of MAP

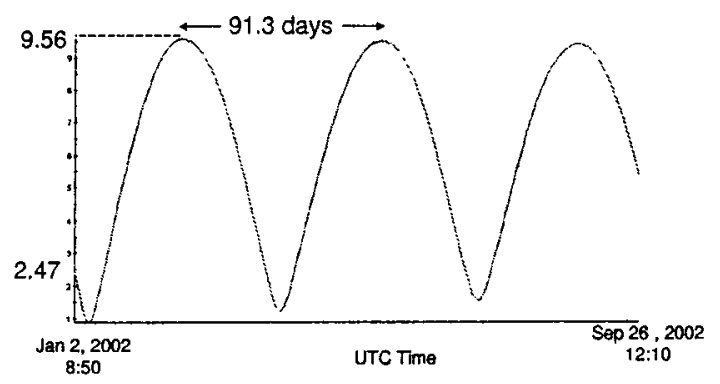

Figure 7: Predicted SEV of MAP (9 Months)

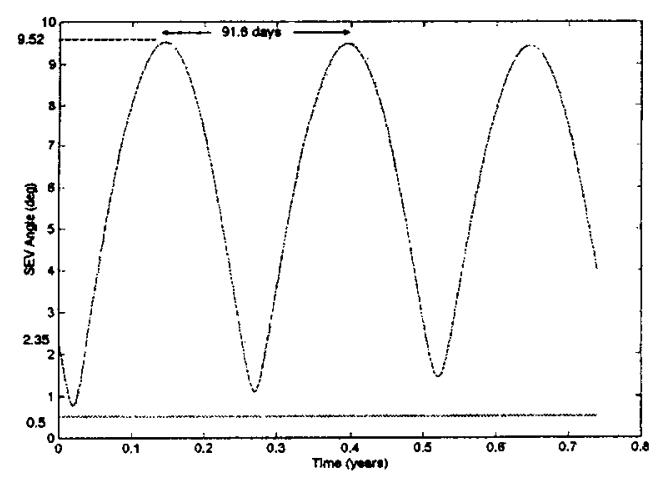

\section{References}

[1] A. Penzias and R. Wilson, Astrophys. J. 142, 419 (1965).

[2] COBE Home Page: http://space.gsfc.nasa.gov /astro/cobe/.

[3] MAP Home Page: http://map.gsfc.nasa.gov/.

[4] V. Szebehely, Theory of Orbits, The Restricted Problem of Three Bodies, New York, Academic Press, 1967.

[5] A.E. Roy, Orbital Motion, Institute of Physics Publishing, London, 1988.

[6] R.W. Farquar, "The Control and Use of Libration-Point Satellites", NASA TR R-346, 1970.

[7] JPL Solar System Dynamics Group, Solar System Dynamics Web page: http://ssd.jpl.nasa.gov/.

[8] D.W. Dunham and D.P. Muhonen, "Tables of Libration-Point Parameters for Selected Solar System Objects," ,Journal of the Astronautical Sciences, Vol. 49, No, 1, January-March 2001, pp.197-217. 